\title{
Reflections about the cranial nerves destinated to the face: plea for a global approach including nerves and their branches, canals, vessels, distribution and clinical relevance
}

\author{
Johann Peltier · Bruno Grignon · Hee-Jin Kim • \\ Jean-Michel Prades · Stéphane Velut · Fabrice Duparc
}

Published online: 18 October 2013

(C) Springer-Verlag France 2013

Cranial nerves I, II, V and VII are destinated to the face and carry sensory and motor fibers. They are usually presented through a neuro-anatomical aspect (true origin, appearing origin, course, distribution), and our purpose was to emphasize the interest of a more global approach with giving interest to the canals, the accompanying vessels, the variations in course and distribution, and the clinical relevance. The importance of the depiction of anatomy had been previously supported [1], and we propose in this issue to emphasize the interest of the imaging of the structures

J. Peltier

Laboratoire d'Anatomie et d'Organogénèse, Faculté de Médecine d'Amiens, Amiens University, Rue des Louvels, 80036 Amiens, France

\section{B. Grignon}

Faculté de Médecine de Nancy, Département d'Anatomie, Université de Lorraine, Nancy University, 9 avenue de la Forêt de Haye, 54505 Vandoeuvre-Les-Nancy, France

\section{H.-J. Kim}

Division in Anatomy and Developmental Biology, Department of Oral Biology, Yonsei University College of Dentistry, Yonsei University, 50 Yonsei-Ro, Seodaemun-Gu, Seoul 120-752, Korea

\section{J.-M. Prades}

Laboratoire d'Anatomie, Faculté de Médecine, Saint-Etienne

University, 15 rue Ambroise Paré, 42023 Saint-Étienne, France

S. Velut

Laboratoire d'Anatomie, Faculté de Médecine de Tours,

Tours University, 10 boulevard Tonnelé, 37032 Tours, France

\section{F. Duparc ( $\square)$}

Laboratoire d'Anatomie, Faculté de Médecine de Rouen, Rouen University, 22 Boulevard Gambetta, 76183 Rouen, France

e-mail: fabrice.duparc@univ-rouen.fr that are surrounding or accompanying these nerves into the face.

This global approach may also be justified by the reciprocal impact of the nerves growth, with great effect about the bone growth. Moreover, the embryology supports this parallel development of nerves, bones and vessels, and the importance of the deflexion of the craniocervical junction after birth must also be considered.

Nerves I and II are specific sensorial cranial nerves, but nerves V and VII are sensory motor nerves, with nuclei originating from branchial arches 1 and 2, respectively. The true origins (within the brain or brainstem) and the appearing origins (the site where the nerves get out of the brain or brainstem) are not concerned in this compilation of articles, or the courses of the trunks and the ganglia in the cranial cavity. The recent techniques of medical imaging have strongly improved the depiction of the nerves and of their main branches, but the course of the ending branches can present important variations, and may be determined through the visualization of the bone canals they run through, and with evaluation of the accompanying vessels.

Two aspects of the course of the olfactory nerve are presented, related to the aspect of the cribriform plates the branches will cross and to the possibilities of variations of the olfactory artery. An unusual position of the ophthalmic artery is presented that may go through the superior orbital fissure but not through the optic canal with the optic nerve, and with origin from a branch of the external carotid artery. Distal branches of the Maxillary Nerve V2 and Mandibular Nerve V3 will be evoked with the courses through the canals and their ending zones.

The intensive improvements of the plastic surgery lead to a great number of researches and publications in the field of applied and clinical anatomy. We have associated in this issue one article about the innervations of the Corrugator 
Supercilii Muscle, at the limit of the face, and another one about the possibility of a venous pedicled temporal flap. The terminating muscle innervations and its blocks and the dissection of vascular and nervous pedicles are technical aspects based on anatomical features.

In this issue will be associated different types of articles, reviews, original articles, presentation of anatomical variations, which are related to the osseous structures that are crossed by the nerves, canals and foramina, to the vessels that are closed to the nerves, with great attention given to the various possibilities of imaging for proposing to the anatomist and the clinician the best conditions of evaluation of these nerves, and safe therapeutic endovascular and surgical procedures.

\section{Reference}

1. Grignon B (2012) Anatomy and medical imaging: a symbiotic relationship. Surg Radiol Anat 34:673-674 